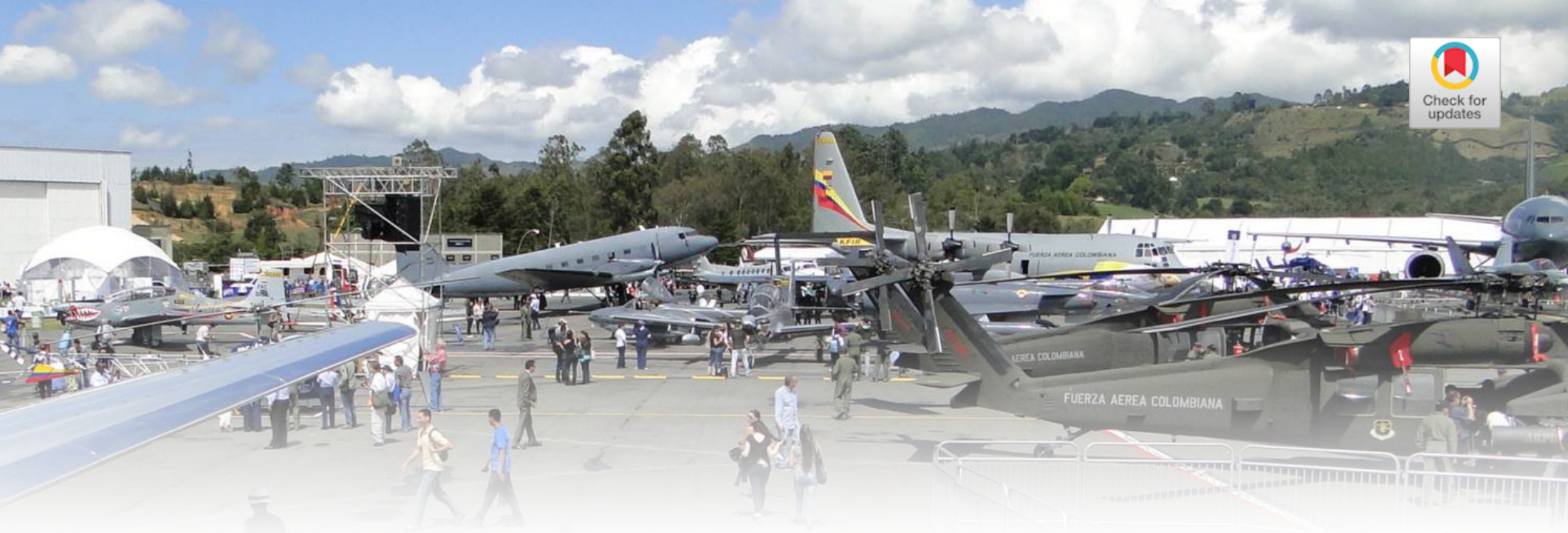

\title{
Apuntes sobre globalización: aspectos culturales, tecnológicos y económicos
}

\author{
Notes on Globalization: Cultural, Technological, and Economic Aspects ${ }^{2}$
}

Mayden Solano Jiménez ${ }^{3}$

CIENCIA Y PODER AÉREO

ISSN 1909-7050 |E-ISSN 2389-2468

Volumen 9 | Enero-Diciembre de 2014 |

Colombia | Pp. 143-150

Recibido: 05/08/2013

Aprobado evaluador interno: 17/08/2013

Aprobado evaluador externo: 26/08/2013

Artículo de reflexión producto de los debates generados en el marco de la línea de investigación de Regionalización y Procesos de Integración.

${ }^{2}$ This reflection article is product of the debates generated through the line of research on Regionalization and Processes of Integration.

${ }^{3}$ Internacionalista y Politóloga, Magíster en Relaciones y Negocios Internacionales de la Universidad Militar Nueva Granada. Profesora e investigadora. Líder del Grupo de Investigación PIREO. Asesora de Investigación de la Escuela de Postgrados de la Fuerza Aérea Colombiana. Actualmente profesora de la Pontificia Universidad Javeriana - Facultad de Ciencias Políticas y Relaciones Internacionales. Correo electrónico: yolisoji@gmail.com

Political Science and International Relations graduate, with a Master degree in International Relations and Business at the Nueva Granada Military University. Professor and researcher. Lead of the Research Group "PIREO". Research advisor at the Graduate School of the Colombian Air Force. She is currently professor at Javeriana University - Political Science and International Relations Department.E-mail:yolisoji@gmail.com
Resumen: La internacionalización de las economías con una consecuente apertura de mercados, la modernización de los organismos tanto públicos como privados, el incremento en la competitividad, las privatizaciones, la reducción del tamaño del Estado y el enlazamiento de las economías; bien por la vía de la regionalización económica de áreas continentales o la integración de mercados en los que intervienen las más disímiles zonas geográficas del mundo; son algunas características propias del reacomodamiento o nuevo orden mundial. Dentro de este contexto, el presente artículo busca analizar el fenómeno de la globalización desde los aspectos: cultural, económico y tecnológico; los cuales han permeado varios sectores de la sociedad global, generando una interrelación e integración entre diversos actores, en donde no sólo confluye el Estado- nación, también da cabida a otros actores del Sistema Internacional. Por lo cual, se analiza el comportamiento de la aldea global y la cultura dentro del fenómeno de la globalización a partir de aspectos económicos y tecnológicos, resaltando la importancia de los medios de comunicación o la llamada era de la información, el mercado financiero global y por último, el papel que juega el proceso de integración y la necesidad de reestructurar las principales instituciones internacionales.

Palabras clave: Globalización cultural, globalización económica, globalización tecnológica, nuevo orden mundial, procesos de integración.

\begin{abstract}
The internationalization of economies with the consequent opening of the markets, the modernization of both public and private organisms; the increase in competitiveness; privatization; reduction in the size of the state; and economic interconnectedness, both by economic regionalization of geographic areas and by the integration of markets in dissimilar geographic regions; are some of the characteristics of the global shift or new world order. Within this context, this article intends to analyze the phenomenon of globalization in its cultural, economic, and technological aspects. These components have permeated various sectors of society on a global level, thus creating integration and interrelations among diverse actors, in which the state does not act alone but it is joined by other actors from the international system. Hence, this article seeks to analyze the behavior of the global village and culture within the phenomenon of globalization, from economic and technological aspects. It highlights the importance of communication media or the so-called information age, the global financial market and, finally, the role played by integrative processes and the need to restructure principal international institutions.
\end{abstract}

Key Words: Cultural Globalization, Economic Globalization, New World Order, Processes of Integration, Technological Globalization. 


\section{Introducción}

La globalización no es un hecho aislado ni tampoco reciente; se relaciona con el desarrollo del capitalismo internacional y con el sistema interestatal, constituye un nuevo momento del proceso de internacionalización. De acuerdo a lo anterior, la globalización hace hincapié en el proceso que elimina las fronteras entre lo interno y lo externo, vinculando varias facetas, es decir que "el proceso de globalización incide en el debilitamiento del EstadoNación en una doble dirección: el carácter mundial que adquieren las actividades económicas, políticas y sociales; la intensificación de las interacciones dentro y entre los estados" (Morales, Rodríguez y Fernández, 1999, p. 33).

El fenómeno de la globalización ha permitido que la economía mundial moderna, con los mecanismos que la integran, es decir, el comercio, la producción y las finanzas, entre otros, inicien espacios de integración cada vez más amplios en el ámbito mundial. En ese sentido, surge como consecuencia de la internacionalización de los procesos económicos, los conflictos sociales y los fenómenos político-culturales.

La globalización está inmersa en el proceso de intercambios de carácter político, económico y cultural. "El anterior proceso es debido a los medios M: Money, Music, Movies, Mathematics, Migration, y Moral Claims" (Claus Off, citado por Salguero, J. s.f., p. 2). Las expresiones culturales se han convertido en el medio por el cual se logra la interconexión de Estados, regiones e individuos, considerando que la cultura se ha establecido como el medio que permite llegar a esa globalización, ya que ofrece identidades de todas partes del mundo, incluso las más apartadas, las cuales hace unos años se desconocían por completo; ahora se sabe de sus valores, imágenes, sus costumbres, creencias, lengua, idioma, es decir, los elementos que identifican a las culturas unas de otras, construyendo una nueva visión del mundo.

La radio, la televisión, la telefonía, el Internet, los discos, los aparatos electrónicos e incluso la música de todas las culturas del planeta, han traspasado fronteras. De esta manera, la cultura se identifica como: "todo lo creado por los seres humanos, la generalidad de la vida de una sociedad, el modo de vida específicamente humano, la totalidad de la experiencia humana acumulada y transmitida socialmente y que en cada grupo humano tiene una concreción y una singularidad" (Ariño, 1997, p. 24). Según lo anterior, la cultura y el proceso de la globalización permiten la conexión de regiones, Estados y demás espacios geográficos.
"La cultura tiende a estar ligada a lugares situados en otros países y en otros continentes" (Hannerz, 1996, p. 33), allí se observa cómo la cultura ha sido un elemento esencial y partícipe en el fenómeno de la globalización, porque no sólo se adquiere conocimiento de una cultura lejana y diferente a la nuestra, sino que sumando a ello se incorporan patrones a la vida cotidiana de las personas y de sus habitad. Un claro ejemplo son los productos que diariamente se encuentran en el mercado de todas partes del mundo, ya no solamente el mercado nacional es el que prima en las tiendas y supermercados, entre otros ejemplos. "Ahora todo lo que se produce está aquí y es difícil saber que es lo propio" (García, 1995, p. 16).

Teniendo en cuenta lo anterior, la globalización cultural es entendida como "un proceso de ensamblado multinacional, una articulación flexible de partes, un montaje de rasgos que cualquier ciudadano de cualquier país, religión o ideología puede leer y usar " (García, 1995, 2012, p. 24). Hoy por hoy, la cultura se ve estrechamente ligada con la apertura de mercados y con la tecnología o los medios de comunicación, que a través de la integración, no solamente de los Estados, también de los nuevos actores internacionales (empresas transnacionales, organismos internacionales, regiones, Sociedad civil, entre otros); facilita la interacción de regiones, de países, formando bloques u organismos de gran envergadura para tratar los problemas del nuevo orden de manera multilateral.

Ahora bien, es necesario avanzar en los siguientes temas para adquirir un acercamiento al comportamiento de la aldea global y la cultura dentro del fenómeno de la globalización a partir de aspectos económicos y tecnológicos. Uno de esos temas es el actuar de los Estados dentro de la comunidad global, teniendo en cuenta la teoría transformacionalista de la globalización. Además, es preciso hablar de la globalización tecnológica, resaltando la importancia de los medios de comunicación o la llamada era de la información. Como tercer tema está el mercado financiero global, y por último, el papel que juega el proceso de integración y la necesidad de reestructurar las principales instituciones internacionales.

\section{La aldea global y la cultura dentro del fenómeno de la globalización}

La Comunidad global se ha regido bajo las siguientes tres tendencias claves para el desarrollo de la globa- 
lización: 1) el cambio tecnológico, 2) el número cada vez mayor de gobiernos que han aplicado políticas de liberación, apertura de mercados, supresión de obstáculos reglamentarios, 3) combinación de nuevas tecnologías y mercados más libres. Estas tres tendencias reflejan el rápido proceso que tiene el mundo para poseer un espacio común en el cual se compartan fuerzas económicas, tecnológicas y sobre todo socio-culturales.

Una de las teorías que permite definir claramente el proceso que la comunidad global está vivenciando, es la teoría transformacionalista ${ }^{4}$; la cual concibe a la globalización como "algo históricamente sin precedentes, de manera que los Estados y las sociedades en todo el planeta experimentan actualmente un proceso de cambio profundo, a medida que tratan de adaptarse a un mundo más interconectado, pero sumamente incierto" (Held y Mcgrew, 2002. p. 31).
Los transformacionalitas rechazan la posición de los hiperglobalistas, en cuanto que manifiestan el fin de la soberanía Estado - Nación. Para la teoría transformacionalista la soberanía, el poder del Estado y la territorialidad, se encuentran hoy en una relación más compleja que en la época durante la cual se forjaba el moderno Estado - Nación, pero eso no quiere decir que haya desaparecido completamente, por el contrario el aparato estatal ha dado cambio a su estructura y a sus funciones debido al modo como los gobiernos buscan estrategias coherentes para insertarse dentro del mundo globalizado (Ver Tabla 1).

\section{Aspectos relevantes de la globalización eco- nómica y tecnológica}

Una de las estrategias que el Estado lleva a cabo es la tecnificación de sus mercados y para ello emplean el recurso tecnológico. Las novedosas máquinas, los

Tabla 1. Conceptualización de la globalización: tres tendencias

\begin{tabular}{|c|c|c|c|}
\hline Variable & Hiperglobalistas & Escépticos & Transformacionalistas \\
\hline ¿Qué hay de nuevo? & Una era global & $\begin{array}{l}\text { Bloques comerciales, un } \\
\text { gobierno territorial más débil } \\
\text { que en períodos anteriores. }\end{array}$ & $\begin{array}{l}\text { Niveles históricamente sin } \\
\text { precedentes de interconexión } \\
\text { global. }\end{array}$ \\
\hline Características dominantes & $\begin{array}{l}\text { Capitalismo global, sociedad } \\
\text { civil global. }\end{array}$ & $\begin{array}{l}\text { Un mundo menos } \\
\text { interdependiente que en la } \\
\text { década de } 1890 .\end{array}$ & $\begin{array}{l}\text { Globalización densa, (intensiva } \\
\text { y extensiva). }\end{array}$ \\
\hline
\end{tabular}

\begin{tabular}{|c|c|c|c|}
\hline $\begin{array}{l}\text { Poder de los gobiernos } \\
\text { nacionales }\end{array}$ & En disminución o erosión. & Reforzado y mejorado. & Reconstituido, reestructurado. \\
\hline $\begin{array}{l}\text { Fuerzas impulsoras de la } \\
\text { globalización }\end{array}$ & El capitalismo y la tecnología. & Estados y mercados. & $\begin{array}{l}\text { Las fuerzas combinadas de la } \\
\text { modernidad. }\end{array}$ \\
\hline Pauta de la estratificación & $\begin{array}{l}\text { La erosión de las antiguas } \\
\text { jerarquías. }\end{array}$ & Creciente marginación del Sur & $\begin{array}{l}\text { r. Nueva arquitectura del orden } \\
\text { mundial. }\end{array}$ \\
\hline Motivo dominante & McDonald's Madonna, etc. & El interés nacional. & $\begin{array}{l}\text { La transformación de la } \\
\text { comunidad política. }\end{array}$ \\
\hline Concepto de globalización & $\begin{array}{l}\text { Como un reordenamiento } \\
\text { de la estructura de la acción } \\
\text { humana. }\end{array}$ & $\begin{array}{l}\text { Como una } \\
\text { internacionalización y una } \\
\text { regionalización. }\end{array}$ & $\begin{array}{l}\text { Como el reordenamiento de } \\
\text { las relaciones interregionales y } \\
\text { de la acción a distancia. }\end{array}$ \\
\hline Trayectoria histórica & Civilización global & $\begin{array}{l}\text { Bloques regionales y choque } \\
\text { de las civilizaciones. }\end{array}$ & $\begin{array}{l}\text { Indeterminada; integración y } \\
\text { fragmentación globales. }\end{array}$ \\
\hline Resumen del argumento & El fin del Estado - nación. & $\begin{array}{l}\text { La internacionalización } \\
\text { depende de la aquiescencia } \\
\text { del apoyo del Estado. }\end{array}$ & $\begin{array}{l}\text { La globalización transforma el } \\
\text { poder del Estado y la política } \\
\text { mundial. }\end{array}$ \\
\hline
\end{tabular}

Fuente: Held, D. y Mcgrew, A. (2002). Transformaciones globales, política, economía y cultura, p. XLI. Oxford: Oxford University Press.

\footnotetext{
${ }^{4}$ Enfoque transformacionalista de la Globalización: sus representantes más reconocidos son: Rosenau y Giddens.
} 
medios de transporte, los medios de comunicación (televisión, radio, Internet) han permitido la enorme interconexión e intercambio entre las diferentes culturas. Anteriormente, era impensable llevar a cabo relaciones de tipo económico, social, cultural y político con Estados o continentes geográficamente distantes; gracias a la diversidad de medios tecnológicos se ha logrado un acercamiento, permitiendo así, una relación entre países del segundo, con el tercer mundo y de estos con países desarrollados.

De acuerdo a lo anterior, se podría concebir la globalización tecnológica como la aplicada en primer lugar en las comunicaciones, pero que se extiende a otras áreas que componen el corazón de las nuevas tecnologías, como la electrónica, los nuevos materiales, la biotecnología, las nuevas fronteras de la química (Katz, 2000). A su vez, la globalización tecnológica tiene relación directa con la competitividad de las empresas, instituciones, organismos e incluso con los entes estatales, donde las "las nuevas máquinas incorporan capacidades operativas que antes eran provistas por personal calificado" (Katz, 2000, p. 13), haciendo que el trabajo y la producción sean óptimos en un tiempo estimado. Lo anterior, promueve cambios en los sistemas de innovación nacional a raíz de las reformas estructurales y del proceso de globalización de la economía.

Uno de los factores importantes que existe en la globalización tecnológica que va sujeta a la parte mercantil ha sido la economía informacional, vital para las empresas, pues de un buen procesamiento de información depende la competitividad y veracidad que arrojan sus datos. Ejemplo: DANE, Registraduría Nacional, Data Crédito, Bolsas de Valores, entre otras. Todas estas entidades gubernamentales o no gubernamentales se valen de un buen procesamiento de información para llevar a cabalidad su función en tan poco tiempo, necesitando de un software o base de datos adaptado a las necesidades de las empresas. Para abastecer este servicio "comienza aparecer un sector doméstico de pequeñas y medianas firmas de ingeniería de sistemas y de producción de software computacional, ocupadas en desarrollar -paquetes informáticos- adaptados a las nuevas necesidades del aparato productivo doméstico" (Katz, 2000, p. 15).

Además, de la economía informacional, está la economía red, "cada vez más las empresas, y todo tipo de organizaciones, funcionan en su práctica cotidiana en redes de relaciones internas" (Castells, 1999a, p. 4). Usan- do la red se permite realizar o desarrollar ciertos estudios al mercado y su adaptación al mismo. Un ejemplo no electrónico es el comprender la importancia de la red y la adquisición de conocimiento, no solamente para el campo comercial.

Benetton vende colores, se llaman los Colores Unidos de Benetton. Partiendo de una pequeña empresa familiar en red al norte de Italia, no una gran empresa sino una multinacional de empresas, se hacen concesiones a un almacén en una ciudad con dos condiciones, vender sólo Benetton y que repartir información. Cada 15 días o cada semana se tomaba la decisión sobre el color que se vende, produciendo una información inmediatamente transmitida al proceso de diseño y luego enviada al sistema de producción operante en red, uniendo la economía informal del sur de Italia y de Turquía. (Castells, 1999a, p. 5).

Según Castells Benetton, es una conexión de información y diseño ligada a múltiples puntos de distribución de mercados y a una producción informal de lo más barato, dado que se trata de ropa deportiva. Lo esencial, es adaptarse a los cambios continuos de color porque en un mismo mundo en donde la gente produce de lo mismo, el color se hace fundamental como elemento de distinción y toque de moda. Manuel Castells en su reseña añade: "recuerdo un ejemplo famoso de planificación soviética; en una ocasión no entendía por qué no se vendían unas camisetas de color amarillo hasta que se dieron cuenta que era el color simbólico del luto" (Castells, 1999a, p. 5).

Con el anterior ejemplo, se confirma la presencia de una economía altamente flexible ligada al funcionamiento en red, dando importancia a las pequeñas y medianas empresas quienes son las que realizan el trabajo de campo, analizan e investigan la cultura a la cual le va ser llevado dicho producto con el objetivo de ser bien acogido por los consumidores locales.

El tema tecnológico sirve de herramienta para llevar a cabo esa interacción o relación de varias culturas, donde la red significa "poner juntos varios elementos, varias personas, varios trozos de empresa o varias empresas para hacer algo juntos, tiene la ventaja de la flexibilidad, de la adaptación rápida a la demanda: cuando hay una demanda fuerte se organiza la red, cuando no la hay, se disuelve y se usan nuevos recursos" (Castells, 1999b, p. 2). 
El mercado financiero se alimenta de una combinación indivisible de comunicación e información, en este ámbito la globalización es vista desde la desregularización y liberación de mercados en todo el mundo. "Este proceso comenzó en Londres más que en los Estados Unidos, exactamente el 27 de Octubre de 1987 mediante el Ilamado Big Bang, cuando se liberaron todos los mercados financieros de esa ciudad" (Castells, 1999a, p. 7). Este acontecimiento permitió la generación de una clase de nuevos productos financieros sintéticos, que fueron combinados con modelos matemáticos en cuales se podría mezclar el valor actual del café, el valor de las acciones del tal empresa, el valor del cambio del yen con el dólar, en fin, un sin número de valores tanto a presente como a futuro, que a su vez se combinan en un producto derivado y se vende en el mercado.

La relación que existe entre los mercados financieros y la globalización es su conexión con los medios electrónicos y las continuas relaciones económicas y financieras en las cuales todo el mundo está inmerso. Estos mercados financieros están interconectados de tres formas:

1) Sus productos derivados toman valores de distintos países y por lo tanto suben y bajan en función de lo que ocurra a un producto determinado en un país específico; 2) los circuitos electrónicos de conexión permiten la transferencia de enormes masas de capital en un segundo, a veces en una fracción de segundo, sin ningún control; 3) existe un proceso de certificación financiera en que las empresas, suben y bajan países o empresas según el comportamiento del mercado mundial (Castells, 1999a, p. 8).

El modelo de desarrollo vigente se enmarca en el neoliberalismo que aboga por la desregularización del mercado, las privatizaciones y la mínima intervención del Estado, ante ello, se recalca que el origen del neoliberalismo se remite al concepto de liberalismo como sinónimo de economía de libre mercado. No obstante, los movimientos de capital han ido cobrando importancia a medida que se liberalizaba el sistema financiero internacional y se suprimían los controles sobre los tipos de cambio. Y en ello, colaboran las telecomunicaciones, de las cuales se valen para mover grandes cantidades de dinero por todo el mundo a una velocidad vertiginosa.
Con el incremento del proceso de globalización de mercados financieros, especialmente se crearon instituciones financieras internacionales más exactamente con el surgimiento de la hegemonía de los Estados Unidos (Plan Marshall) con el objetivo de regular la globalización del comercio y la liberación económica. No solamente se centró en la tarea de crear espacios internacionales para deliberaciones con respecto al comercio y la economía mundial, además, se empezó a hablar de Integración ${ }^{5}$.

Los acuerdos comerciales se han incrementado en las últimas décadas, permitiendo así una integración económica definida como el "proceso a través del cual se eliminan las fronteras económicas entre países. Estas fronteras se asocian con las barreras al comercio, con discriminación entre economías nacionales, con varios obstáculos que frenan una verdadera integración de Estados, de Regiones y demás actores internacionales. A la par de una integración económica vienen integraciones de tipo social, político, cultural que buscan la paz y el fomento de la democracia. De ahí que se contempla el Desarrollo del milenio, cuyo objetivo es reducir a la mitad la pobreza y el hambre del mundo al 2015, cubrir el tema de la salud, combatir la Malaria, el SIDA, temas tales como desarrollo sostenible y cambio climático, entre otros.

\section{La necesidad de reestructurar las principa- les instituciones internacionales e integra- ción de nuevos actores}

Para los temas de gran envergadura de tipo social, político y económico se han llevado a cabo integraciones regionales bajo los criterios de vecindad, afinidad tanto cultural, como económica. De ésta manera, el regionalismo estaría ligado a iniciativas políticas y económicas entre Estados que hacen parte de una misma región. Dentro de las formas de integración de tipo económico se destacan los acuerdos preferenciales, la zona de libre comercio, la unión aduanera, mercado común o mercado único, unión económica y unión monetaria.

Para muchos países resulta difícil llevar a cabo el proceso de integración con sus vecinos o con países industrializados. Según los transformacionalitas "la globalización está asociada con nuevas pautas de estratificación en las que algunos Estados, sociedades y co-

${ }^{5}$ Integración viene del vocablo "Integratio" que significa adhesión, unión de partes. Diccionario de la Real Academia. 
munidades, se interconectan cada vez más en el orden global, mientras que otros se vuelven cada vez más marginados" (Held et al, 2002, p. 38).

Entonces, la liberación del comercio y la globalización traen consigo varios desafíos de los que se encuentran: 1) consecuencias en la distribución de las rentas; pues hay quienes ganan y quienes pierden y no todos participan plenamente en los beneficios de la globalización, 2) fomentar el crecimiento, el desarrollo en el proceso de globalización, pueden causarse daños al medio ambiente. 3) las fuerzas del mercado en cierta manera obligan a adoptar a los países normas más bajas para competir, llegando a debilitar a los gobiernos nacionales, siendo estos menos eficaces para cumplir sus deberes económicos y sociales. Para resolver los problemas que traen el comercio y la liberación se necesita de organismos internacionales financieros que permitan la regularización de estos procesos. Dentro de las instituciones se reconocen el $\mathrm{FMI}^{\mathrm{B}} \mathrm{BM}^{6}$.

Estas instituciones financieras u organismo internacionales financieros deberán reformar sus estructuras acorde con la agenda internacional actual."La economía mundial y el sistema financiero internacional son muy diferentes ahora de lo que se contempló en la conferencia de Bretton Woods, en 1944, cuando fueron establecidos el Fondo Monetario Internacional y el Banco Mundial" (Meltzer, 2001, p. 9).

Allan Meltzer, catedrático de economía política y política pública - Universidad Carnegie Mellon, propone algunos cambios estructurales necesarios dentro de los cuales se mencionan los siguientes: a) La función apropiada del FMI debe ser la de prevenir las crisis financieras y prevenir la propagación de aquellas crisis que ocurren, b) El FMI debe asesorar, pero no debe condicionar el asesoramiento a la ayuda. c) El FMI necesita concentrarse en cuatro tareas principales: la prevención de crisis,

\footnotetext{
${ }^{6}$ La misión del FMI consistía en supervisar y regular el sistema monetario mundial y mantener el equilibrio y la estabilidad monetaria internacional, luego se incursiona en el ámbito de desarrollo que junto con el apoyo del BM se orientan en el diseño, financiación y seguimiento de los planes de Ajuste Estructural PEA: Orientados a restablecer el equilibrio en la Balanza de pagos de los países afectados por la crisis para permitir que cumpliesen con el servicio de la deuda. Hoy por hoy (2013), su misión es promover la estabilidad financiera y la cooperación monetaria internacional. Asimismo, busca facilitar el comercio internacional, promover un empleo elevado y un crecimiento económico sostenible y reducir la pobreza en el mundo entero. Fundado en 1945, es administrado por los 188 países miembros — casi todos los países del mundo_ a los cuales les rinde cuentas. Página oficial del FMI www.imf.org.
}

el manejo de las crisis, mejorar la calidad y aumentar la cantidad de la información pública, y proveer asesoramiento macroeconómico a los países en desarrollo.

No todas las crisis pueden prevenirse. Sin embargo, se puede reducir la frecuencia y gravedad de las crisis reformando las prácticas del país y del FMl con el fin de aumentar los incentivos para adoptar políticas y conductas que acrecienten la estabilidad (Meltzer, 2001, p. 9).

En cuanto a la misión de los bancos de desarrollo, el Banco Mundial y los bancos regionales de desarrollo deberían: a) mejorar la calidad de vida, reducir la pobreza, y proveer bienes públicos globales y regionales; es decir, promover el desarrollo, no los préstamos; b) seguir proveyendo asistencia técnica y fomentar la transferencia de conocimientos en todas las regiones; c) deben trabajar para mejorar la calidad de la vida, aún en los países donde la corrupción y los arreglos institucionales obstaculicen o impidan el desarrollo económico; d) encontrar soluciones para los problemas comunes, entre los que figuran asuntos de salud, pobreza, cambio climático y muchas otras áreas.

En un mundo cada vez más globalizado se necesita reformar las instituciones financieras internacionales para aumentar la estabilidad económica, mejorar el movimiento de información, estimular el desarrollo económico, reducir la pobreza, apoyar la provisión de bienes públicos regionales y globales e incrementar la participación de la microempresas, sin caer en la distribución cada vez más desigual en el mundo. Abriendo espacio a una integración diversificada o de un interregionalismo contemporáneo, el cual responde a tres topologías: 1) Relaciones grupo a grupo, 2) acuerdos transregionales; 3) relaciones entre grupos y potencias regionales.

Los anteriores, son elementos de análisis que podrían trabajarse en primera instancia desde el análisis del neoinstitucionalismo y lo que se enmarca en relación al concepto de multilateralismo; por mencionar otros enfoques se incluye regionalismo abierto o nuevo regionalismo. El neoinstitucionalismo se presenta en respuesta al estudio de la complejidad en las instituciones ante el papel que éstas desempeñan en la vida política, social y económica dentro del nuevo orden mundial, donde las instituciones no son estáticas, estas deben "hallar la manera de adaptarse a los cambios de sus entornos, lo que significaría que las instituciones se deben adecuar mediante el fortalecimiento de sus áreas de acción a tra- 
vés de su cambio institucional" (Molina, Beltrán y Solano, 2012, p. 84); por lo que no son muchas las Instituciones que permanecen actualmente, o por lo menos van en detrimento de sus políticas, siendo rígidas e inertes antes los cambios generados por las aperturas a los mercados, trasferencia de tecnología, por las TIC, aspectos de multiculturalidad y demás consecuencias derivadas de la globalización.

Por otra parte, el multilateralismo permite generar relaciones de tipo económico, social, político y medioambiental, con actores diversos "El objetivo del multilateralismo es integrar a todos los Estados en una misma comunidad de reglas" (Moreau, 2004, p. 4). Este concepto ha sido abordado desde la disciplina de las Relaciones Internacionales, a diferencia de procesos de integración bilateral, que sin escatimar su importancia, por ejemplo, para el escenario de los acuerdos comerciales, se han promovido con mayor esfuerzo desde un enfoque de integración regional, donde no sólo se invita a participar a los Estados, también se involucra a otros actores, que antes no eran contemplados dentro de las estructuras institucionales, adquiriendo relevancia en la toma de decisiones, en la puesta en marcha de políticas y acciones que permitan cumplir con los retos previamente establecidos en escenarios multilaterales de una agenda común.

Para los globalizadores, el sistema internacional va incorporado nuevos actores que desafían en menor o mayor grado, la supremacía del Estado como actor; tal es el caso de las organizaciones internacionales, las ONG, las empresas transnacionales, que han originado que el Estado se convierta en un campo de batalla fragmentado de ejecución de políticas, penetrado por redes transaccionales (gubernamentales y no gubernamentales) así como por instituciones de fuerzas nacionales. Del mismo modo, la amplia penetración de la sociedad civil de fuerzas transnacionales ha alterado su forma y dinámica (Held et al, 2003, p. 29).

\section{Conclusiones}

La globalización es un proceso continuamente dinámico y sujeto a cambios, no solamente en la esfera económica, sino política, social y cultural; unos de sus principales síntomas, haciendo alusión a las principales manifestaciones del proceso que ha desembocado la apertura de los mercados y ha generado un mayor comercio internacional, se citan a continuación, aclarando que no son los más relevantes: 1) expansión del comercio de bienes y servicios; 2) cambio de los actores (mercados emergentes como Brasil, Rusia, India, China; 3) creciente presencia de las empresas multinacionales y con estos otros actores; 4) dispersión de la producción mundial a través del progreso tecnológico sumado a las tecnologías de la información y las comunicaciones; 5) Integración financiera. También, es importante citar los desarreglos ecológicos o cambios en los ecosistemas, degradación del medio ambiente a causa de la desaforada apertura de mercados, consumismo que se inserta en las nuevas dinámicas de las sociedades, sin atender los límites que ello genera, en especial para el medio ambiente.

Las nuevas tecnologías siempre han jugado un papel crucial en los procesos de globalización económica y cultural, han hecho posible la comercialización de bienes y contactos individuales a través del globo. Pues hay quienes afirman que la globalización actual sería imposible sin tales tecnologías.

El fenómeno de la globalización trae consigo le necesidad de cambio en las dinámicas de interrelación entre actores, donde no sólo son los Estados, actores por excelencia, también hay participación de otros en distintos niveles de poder o inherencia, los cuales son estimados por sus propuestas y experiencias, cuyas acciones se hacen evidentes en escenarios donde se discuten temas de agenda común a nivel local, regional y del globo, teniendo realmente una cohesión social ante la diversidad. El cambio propuesto no sólo está enmarcado a nivel de stakeholders, también a nivel de estructuras organizacionales. Las instituciones ya no deben ser las mismas de hace algunos años, éstas deben generar cambios que les permitan permanecer en el tiempo; transformaciones a nivel tanto de políticas, como de acciones, así como explorar nuevas dinámicas de integración, a nivel regional, interregional y mundial.

Las interrelaciones entre actores a distintos niveles, permite abrir el debate de lo que se conoce como gobernanza global y gobernanza multinivel, en el marco de la mundialización, producto de la globalización como detonante de cambios y nuevas dinámicas culturales que permean varios escenarios y sectores. La gobernanza busca generar bases que contribuyen a acciones colectivas a partir de los problemas catalogados como comunes - globales, o incluso de carácter regional, que necesitan ser resueltos o atendidos y que los Estados, instituciones y demás grupos de actores por sí solos les resulta difícil resolver, dada la poca interacción entre ac- 
CIENCIA Y PODER AÉREO | Revista Científica de la Escuela de Postgrados de la Fuerza Aérea Colombiana | Vol. 9 | Enero - Diciembre de 2014

tores desde el actor local hasta llegar a la red mundial. Este es el gran reto del Sistema Internacional, y con ello nuevas dinámicas de participación de los stakeholders; temas que esperan ser analizados en siguientes escritos.

\section{Referencias}

Ariño, V. A. (1997). Sociología de la cultura. Barcelona: Editorial Ariel.

Castells, M. (Mayo, 1999a). Globalización, sociedad y política en la era de la información. [Ponencia] Auditorio León de Greiff. Bogotá: Universidad Nacional de Colombia.

Castells, M. (1999b). Capítulo 1. La transformación del trabajo. Globalización, tecnología, trabajo, empleo y empresa. [En línea] Disponible en http://www.revistalafactoria.eu/articulo. php?id=102 Condultado el 24 de agosto de 2013.

García, C. N. (1995). Introducción: consumidores del siglo XXI, ciudadanos del XVIII. En Consumidores y ciudadanos. México: Grijalbo.

García, C. N. (2012). Consumidores y ciudadanos: Conflictos multiculturales de la globalización. México: Grijalbo.

Hannerz, U. (1996). Lo local y lo global: continuidad y cambio. En: Gomis, M. (trad). Conexiones transnacionales: cultura, gente, lugares. España: Ediciones Cátedra S.A.

Held, D. y Mcgrew, A. (2002). Transformaciones globales: política, economía y cultura. Harla, México: Oxford University Press

Held, D. y Mcgrew, A. (2003). Globalización / Antiglobalización: sobre la reconstrucción del orden mundial. Barcelona: Paidós América S.A.

Meltzer, A. H. (2001). Las reformas de las Instituciones Financieras Internacionales: un plan para la estabilidad financiera y el desarrollo económico. Perspectivas Económicas 6 (1).pp. 9-12.

Molina, A. M., Beltrán, P. P. y Solano, M. Y. (2012). Importancia de Evaluar las funciones de la organización interestatal regional frente a los temas de desarrollo sostenible: caso OEA. [Ponencia] I Simposio Interinstitucional de Investigaciones Piloto - Lanzamiento Cátedra Latinoamericana y Caribeña de Integración 2011 - Diálogos desde la sociedad civil para la Integración. Bogotá, Colombia: Universidad Piloto de Colombia, Asociación de Universidades de América Latina y el Caribe para la Integración - AUALCPI.

Moreau Defarges, P. (2004). Le multilatéralisme et la fin de I'histoire. Revue politique étrangére, automne. En Rueda Montreal, R. (trad.). El multilateralismo y el fin de la historia.

Morales Manzur, J., Rodríguez Sangroni, H. y Fernández, M. A. (Mayo - Octubre, 1999). La cultura de la integración latinoamericana en la globalización: el papel de los medios. Aldea mundo (7). Mérida, Venezuela: Biblioteca Digital Andina, Universidad de los Andes.

Salguero Cubides, J. (s.f.). Globalización, economía y regiones de Colombia. Sociedad Geográfica de Colombia. Colombia: Academia de Ciencias Geográficas.

Katz, J. (2000). Globalización, Reformas Estructurales y Sistemas Innovativos. Desarrollo productivo № 75. Santiago de Chile: CEPAL.

Para citar este artículo Solano, M. (2014). Apuntes sobre globalización: aspectos culturales, tecnológicos y económicos. Ciencia y Poder Aéreo, Vol. 9 (1). Pp. 143-150 